\title{
SYMBOLIC DYNAMICS IN MEAN DIMENSION THEORY
}

\author{
MAO SHINODA, MASAKI TSUKAMOTO
}

\begin{abstract}
Furstenberg (1967) calculated the Hausdorff and Minkowski dimensions of one-sided subshifts in terms of topological entropy. We generalize this to $\mathbb{Z}^{2}$-subshifts. Our generalization involves mean dimension theory. We calculate the metric mean dimension and mean Hausdorff dimension of $\mathbb{Z}^{2}$-subshifts with respect to a subaction of $\mathbb{Z}$. The resulting formula is quite analogous to Furstenberg's theorem. We also calculate the rate distortion dimension of $\mathbb{Z}^{2}$-subshifts in terms of Kolmogorov-Sinai entropy.
\end{abstract}

\section{INTRODUCTION}

1.1. Hausdorff and Minkowski dimensions of subshifts. Let $A$ be a finite set (alphabet). We consider the one-sided infinite product $A^{\mathbb{N}}=A \times A \times A \times \cdots$ with the shift map $\sigma: A^{\mathbb{N}} \rightarrow A^{\mathbb{N}}$ defined by

$$
\sigma\left(\left(x_{n}\right)_{n \in \mathbb{N}}\right)=\left(x_{n+1}\right)_{n \in \mathbb{N}} .
$$

Take $\alpha>1$. We define a distance $d$ on $A^{\mathbb{N}}$ by

$$
d(x, y)=\alpha^{-\min \left\{n \mid x_{n} \neq y_{n}\right\}} .
$$

Let $\mathcal{X} \subset A^{\mathbb{N}}$ be a $\sigma$-invariant closed subset. Furstenberg [Fur67, Proposition III.1] calculated the Hausdorff and Minkowski dimensions of $\mathcal{X}$ with respect to $d$ :

$$
\operatorname{dim}_{\mathrm{H}}(\mathcal{X}, d)=\operatorname{dim}_{\mathrm{M}}(\mathcal{X}, d)=\frac{h_{\text {top }}(\mathcal{X}, \sigma)}{\log \alpha} .
$$

Here $h_{\text {top }}(\mathcal{X}, \sigma)$ is the topological entropy of $(\mathcal{X}, \sigma)$. The purpose of the paper is to extend this result to higher rank actions.

1.2. Mean dimension theory. Mean dimension theory provides a meaningful framework for extending (1.1) to higher rank actions. This is the theory first introduced by Gromov [Gro99] and further developed by Lindenstrauss-Weiss [LW00], Lindenstrauss [Lin99], and more recently Lindenstrauss and the second named author [LT19]. We review the basic ingredients here. (The precise definitions will be given in $§ 2$.)

Date: October 3, 2019.

2010 Mathematics Subject Classification. 37A05, 37B10, 37C45, 94A34.

Key words and phrases. subshift, metric mean dimension, mean Hausdorff dimension, rate distortion dimension.

M.S. was partially supported by Grant-in-Aid for JSPS Research Fellow, JSPS KAKENHI Grant Number 17J03495. M.T. was partially supported by JSPS KAKENHI 18K03275. 
A pair $(\mathcal{X}, T)$ is called a dynamical system if $\mathcal{X}$ is a compact metric space and $T$ : $\mathcal{X} \rightarrow \mathcal{X}$ is a homeomorphism ${ }^{1}$. Gromov [Gro99] defined mean topological dimension $\operatorname{mim}(\mathcal{X}, T)$. This is a dynamical analogue of topological dimension, and it evaluates the number of parameters per iterate for describing the orbits of $(\mathcal{X}, T)$. As the name suggested, the mean topological dimension is a topological invariant of dynamical systems. There are many important works around this quantity [LW00, Lin99, Gut15, GLT16, GT, LL18, Tsu18, MT19, LT19]. However mean topological dimension is not the right notion for the purpose of this paper because Furstenberg's theorem (1.1) concerns with Hausdorff and Minkowski dimensions, not topological one. (The topological dimension of a subshift $\mathcal{X} \subset A^{\mathbb{N}}$ is simply zero.)

Let $d$ be a metric (i.e. a distance function) on $\mathcal{X}$. Lindenstrauss-Weiss [LW00] defined metric mean dimension $\operatorname{mdim}_{\mathrm{M}}(\mathcal{X}, T, d)$. This is a dynamical analogue of Minkowski dimension. Lindenstrauss and the second named author [LT19] defined mean Haus-

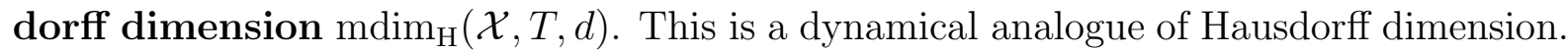
Metric mean dimension and mean Hausdorff dimension are metric dependent quantities. They provide a good framework for the purpose of the paper.

It is well-known in geometric measure theory [Mat95] that metrical dimensions are deeply connected to measure theory. In particular we can introduce the concept of (metric dependent) dimension for measure (see e.g. [Rén59, Youn82, KD94]). Similarly we can introduce a mean dimensional quantity for invariant measures of dynamical systems. Let $\mu$ be a $T$-invariant Borel probability measure on $\mathcal{X}$. Let $X$ be a random variable taking values in $\mathcal{X}$ according to the law $\mu$, and we consider the stochastic process $\left\{T^{n} X\right\}_{n \in \mathbb{Z}}$. We denote by $R(d, \mu, \varepsilon)(\varepsilon>0)$ the rate distortion function of this stochastic process. This is the key quantity of Shannon's rate distortion theory [Sh48, Sh59]. It evaluates how many bits per iterate we need for describing the process within the distortion (with respect to $d$ ) bound by $\varepsilon$. Following Kawabata-Dembo [KD94], we define the upper and lower rate distortion dimensions $b^{2}$

$$
\overline{\operatorname{rdim}}(\mathcal{X}, T, d, \mu)=\limsup _{\varepsilon \rightarrow 0} \frac{R(d, \mu, \varepsilon)}{\log (1 / \varepsilon)}, \quad \underline{\operatorname{rdim}}(\mathcal{X}, T, d, \mu)=\liminf _{\varepsilon \rightarrow 0} \frac{R(d, \mu, \varepsilon)}{\log (1 / \varepsilon)} .
$$

When the upper and lower limits coincide, we denote the common value by $\operatorname{rdim}(\mathcal{X}, T, d, \mu)$.

Metric mean dimension, mean Hausdorff dimension and rate distortion dimension are related to each other. See Proposition 2.1 and Theorem 2.4 below.

1.3. Statement of the main result. Let $A$ be a finite set as in $\S 1$. We consider the infinite product $A^{\mathbb{Z}^{2}}$ index by $\mathbb{Z}^{2}$. We define the shifts $\sigma_{1}$ and $\sigma_{2}$ on $A^{\mathbb{Z}^{2}}$ by

$$
\sigma_{1}\left(\left(x_{m, n}\right)_{m, n \in \mathbb{Z}}\right)=\left(x_{m+1, n}\right)_{m, n \in \mathbb{Z}}, \quad \sigma_{2}\left(\left(x_{m, n}\right)_{m, n \in \mathbb{Z}}\right)=\left(x_{m, n+1}\right)_{m, n \in \mathbb{Z}} .
$$

\footnotetext{
${ }^{1}$ We can also consider a non-invertible map $T$ as in $\S 1.1$. But we consider only invertible $T$ here for simplicity.

${ }^{2}$ Throughout the paper, we assume that the base of the logarithm is two.
} 
Fix $\alpha>1$ and define a distance $d$ on $A^{\mathbb{Z}^{2}}$ by

$$
d(x, y)=\alpha^{-\min \left\{|u|_{\infty} \mid x_{u} \neq y_{u}\right\}},
$$

where $|u|_{\infty}=\max (|m|,|n|)$ for $u=(m, n) \in \mathbb{Z}^{2}$. We call a closed subset $\mathcal{X} \subset A^{\mathbb{Z}^{2}}$ subshift if it is invariant under both $\sigma_{1}$ and $\sigma_{2}$.

The following is our main result.

Theorem 1.1. Let $\mathcal{X} \subset A^{\mathbb{Z}^{2}}$ be a subshift. Then

$$
\operatorname{mdim}_{\mathrm{H}}\left(\mathcal{X}, \sigma_{1}, d\right)=\operatorname{mdim}_{\mathrm{M}}\left(\mathcal{X}, \sigma_{1}, d\right)=\frac{2 h_{\mathrm{top}}\left(\mathcal{X}, \sigma_{1}, \sigma_{2}\right)}{\log \alpha}
$$

Here $h_{\text {top }}\left(\mathcal{X}, \sigma_{1}, \sigma_{2}\right)$ is the topological entropy of $\left(\mathcal{X}, \sigma_{1}, \sigma_{2}\right)$. Moreover, if $\mu$ is a Borel probability measure on $\mathcal{X}$ invariant under both $\sigma_{1}$ and $\sigma_{2}$ then

$$
\operatorname{rdim}\left(\mathcal{X}, \sigma_{1}, d, \mu\right)=\frac{2 h_{\mu}\left(\mathcal{X}, \sigma_{1}, \sigma_{2}\right)}{\log \alpha}
$$

Here $h_{\mu}\left(\mathcal{X}, \sigma_{1}, \sigma_{2}\right)$ is the Kolmogorov-Sinai entropy of $\left(\mathcal{X}, \sigma_{1}, \sigma_{2}\right)$ with respect to the measure $\mu$.

In particular if $\mu$ is a maximal entropy measure (i.e. $h_{\mu}\left(\mathcal{X}, \sigma_{1}, \sigma_{2}\right)=h_{\text {top }}\left(\mathcal{X}, \sigma_{1}, \sigma_{2}\right)$ ) then $\operatorname{rdim}\left(\mathcal{X}, \sigma_{1}, d, \mu\right)$ coincides with the mean Hausdorff dimension and metric mean dimension.

The point of the statement is that we consider various mean dimensional quantities for the action of $\sigma_{1}$, not the total $\mathbb{Z}^{2}$-action generated by $\sigma_{1}$ and $\sigma_{2}$. In other words we consider only $\sigma_{1}$ and disregard $\sigma_{2}$. Nevertheless we can recover the entropy of the total $\mathbb{Z}^{2}$-action. This might look a bit strange at first sight. But in fact it has the same spirit with Furstenberg's theorem (1.1). In (1.1), we consider the Hausdorff and Minkowski dimensions of one-sided subshifts. Hausdorff and Minkowski dimensions are purely metric invariants and do not involve dynamics. So here we disregard the action at all. However we can recover the topological entropy. See Remark 1.2 (4) below for more backgrounds behind the formulation of the theorem.

Remark 1.2. (1) Subshifts $\mathcal{X} \subset A^{\mathbb{Z}^{2}}$ are totally disconnected. So the mean topological dimension of $\left(\mathcal{X}, \sigma_{1}\right)$ is zero.

(2) Probably some readers notice a slight difference between Furstenberg's theorem (1.1) and our (1.4): Our formula involves the coefficient "2" wheres Furstenberg's theorem does not. This difference comes from the point that Furstenberg's theorem considers one-sided shifts (i.e. actions of $\mathbb{N}$, not $\mathbb{Z}$ ). If we consider two-sided shifts, then we get a result completely analogous to (1.4).

(3) Theorem 1.1 can be generalized to $\mathbb{Z}^{k}$-shifts and, probably some noncommutative group actions. But we stick to $\mathbb{Z}^{2}$ for simplicity of the exposition. 
(4) A guiding principle behind our theorem is as follows: Let $T: \mathbb{Z}^{k} \times \mathcal{X} \rightarrow \mathcal{X}$ be a continuous action of $\mathbb{Z}^{k}$ on a compact metric space $\mathcal{X}$. If $T$ has some hyperbolicity-like property, then we can control the mean dimensional quantities of the restriction of $T$ to subgroups $G \subset \mathbb{Z}^{k}$ with $\operatorname{rank} G=k-1$.

When $k=1$, the subgroup $G$ must be trivial. So, in particular, this principle claims that we can control the dimensions of $\mathcal{X}$ if $\mathcal{X}$ admits an action of $\mathbb{Z}$ with some "hyperbolicty". Furstenberg's theorem (1.1) is a typical example of such results because symbolic dynamics can be seen as an extreme case of hyperbolic dynamics. Theorem (1.1) corresponds to the case of $k=2$ in this principle.

Another manifestation of the above principle was given by the work of [MT19]. They proved that if $T: \mathbb{Z}^{k} \times \mathcal{X} \rightarrow \mathcal{X}$ is expansive then the mean topological dimension of $\left.T\right|_{G}$ is finite for any rank $(k-1)$ subgroups $G \subset \mathbb{Z}^{k}$. In particular, when $k=1$, a compact metric space has finite topological dimension if it admits an expansive action of $\mathbb{Z}$. This is a classical theorem of Mañé [Ma79].

(5) We consider the action of $\sigma_{1}$ in Theorem 1.1. This corresponds to a study of the action of the subgroup $\{(n, 0) \mid n \in \mathbb{Z}\} \subset \mathbb{Z}^{2}$. According to the principle in the above (4), it is also natural to consider other rank-one subgroups. Namely we should study various mean dimensional quantities for the action of $\sigma_{1}^{a} \sigma_{2}^{b}$ for any nonzero $(a, b) \in \mathbb{Z}^{2}$, which corresponds to the subgroup $\{(a n, b n) \mid n \in \mathbb{Z}\}$.

Indeed we can calculate them. Take a nonzero $(a, b) \in \mathbb{Z}^{2}$. Then for a subshift $\mathcal{X} \subset A^{\mathbb{Z}^{2}}$ we have

$$
\begin{aligned}
& \operatorname{mdim}_{\mathrm{M}}\left(\mathcal{X}, \sigma_{1}^{a} \sigma_{2}^{b}, d\right)=\operatorname{mdim}_{\mathrm{H}}\left(\mathcal{X}, \sigma_{1}^{a} \sigma_{2}^{b}, d\right)=2(|a|+|b|) \frac{h_{\mathrm{top}}\left(\mathcal{X}, \sigma_{1}, \sigma_{2}\right)}{\log \alpha} \\
& \operatorname{rdim}\left(\mathcal{X}, \sigma_{1}^{a} \sigma_{2}^{b}, d, \mu\right)=2(|a|+|b|) \frac{h_{\mu}\left(\mathcal{X}, \sigma_{1}, \sigma_{2}\right)}{\log \alpha}
\end{aligned}
$$

Here $d$ is the metric defined by (1.3) and $\mu$ is a Borel probability measure on $\mathcal{X}$ invariant under $\sigma_{1}$ and $\sigma_{2}$.

The factor $2(|a|+|b|)$ in (1.5) has the following geometric meaning. For natural numbers $M$ and $N$, we define $\Lambda_{a, b}(M, N) \subset \mathbb{Z}^{2}$ as the set of points

$$
(a n+x, b n+y), \quad\left(0 \leq n<N,|(x, y)|_{\infty}<M\right) .
$$

Here $n, x, y$ are integers. (Namely, we consider the parallel translations of $(-M, M)^{2}$ along the segment $\{(a n, b n) \mid 0 \leq n<N\}$. ) Then we have

$$
2(|a|+|b|)=\lim _{M \rightarrow \infty}\left(\lim _{N \rightarrow \infty} \frac{\left|\Lambda_{a, b}(M, N)\right|}{M N}\right) \quad(|\cdot| \text { denotes the cardinality }) .
$$

The square $(-M, M)^{2}$ is the disk of radius $M$ in the $\ell^{\infty}$-norm $|u|_{\infty}$. The relevance of the $\ell^{\infty}$-norm here comes from the point that the metric (1.3) uses it. If we use a different metric, then we get a different result. For example, consider the 
following metric $\rho$ on $A^{\mathbb{Z}^{2}}$ :

$$
\rho(x, y)=\alpha^{-\min \left\{\sqrt{m^{2}+n^{2}} \mid x_{m, n} \neq y_{m, n}\right\}} .
$$

This metric uses the $\ell^{2}$-norm $\sqrt{m^{2}+n^{2}}$ instead of the $\ell^{\infty}$-norm. For this metric we have

$$
\begin{aligned}
& \operatorname{mdim}_{\mathrm{M}}\left(\mathcal{X}, \sigma_{1}^{a} \sigma_{2}^{b}, \rho\right)=\operatorname{mdim}_{\mathrm{H}}\left(\mathcal{X}, \sigma_{1}^{a} \sigma_{2}^{b}, \rho\right)=2 \sqrt{a^{2}+b^{2}} \cdot \frac{h_{\text {top }}\left(\mathcal{X}, \sigma_{1}, \sigma_{2}\right)}{\log \alpha}, \\
& \operatorname{rdim}\left(\mathcal{X}, \sigma_{1}^{a} \sigma_{2}^{b}, \rho, \mu\right)=2 \sqrt{a^{2}+b^{2}} \cdot \frac{h_{\mu}\left(\mathcal{X}, \sigma_{1}, \sigma_{2}\right)}{\log \alpha} .
\end{aligned}
$$

The proofs of (1.5) and (1.7) are conceptually the same with the proof of Theorem 1.1. However they become notationally more messy. So we decide to concentrate on the statement of Theorem 1.1. It clarifies the ideas in the simplest form.

Acknowledgment. The first proof we gave to Theorem 1.1 contained a gap. Elon Lindenstrauss pointed out this, and he also kindly explained to us how to fix the gap. We would like to thank him for the help.

\section{Preliminaries}

The purpose of this section is to define the three dynamical dimensions (metric mean dimension, mean Hausdorff dimension and rate distortion dimension $)^{3}$ and explain some of their basic properties.

2.1. Metric mean dimension and mean Hausdorff dimension. Let $(\mathcal{X}, d)$ be a compact metric space. For $\varepsilon>0$ we define $\#(\mathcal{X}, d, \varepsilon)$ as the minimum natural number $n$ such that $\mathcal{X}$ can be covered by open sets $U_{1}, \ldots, U_{n}$ with $\operatorname{diam} U_{i}<\varepsilon$ for all $1 \leq i \leq n$. The upper and lower Minkowski dimensions of $(\mathcal{X}, d)$ are given by

$$
\overline{\operatorname{dim}}_{\mathrm{M}}(\mathcal{X}, d)=\limsup _{\varepsilon \rightarrow 0} \frac{\log \#(\mathcal{X}, d, \varepsilon)}{\log (1 / \varepsilon)}, \quad \underline{\operatorname{dim}}_{\mathrm{M}}(\mathcal{X}, d)=\liminf _{\varepsilon \rightarrow 0} \frac{\log \#(\mathcal{X}, d, \varepsilon)}{\log (1 / \varepsilon)} .
$$

For $s \geq 0$ and $\varepsilon>0$ we define

$$
\mathcal{H}_{\varepsilon}^{s}(\mathcal{X}, d)=\inf \left\{\sum_{i=1}^{\infty}\left(\operatorname{diam} E_{i}\right)^{s} \mid \mathcal{X}=\bigcup_{n=1}^{\infty} E_{i} \text { with diam } E_{i}<\varepsilon \text { for all } i \geq 1\right\} .
$$

Here we use the convention that $0^{0}=1$ and $\operatorname{diam}(\emptyset)^{s}=0$. Since $\mathcal{X}$ is compact, this is equal to the infimum of

$$
\sum_{i=1}^{n}\left(\operatorname{diam} U_{i}\right)^{s}
$$

over all finite open covers $\left\{U_{1}, \ldots, U_{n}\right\}$ of $\mathcal{X}$ with $\operatorname{diam} U_{i}<\varepsilon$ for all $1 \leq i \leq n$. We set

$$
\operatorname{dim}_{\mathrm{H}}(\mathcal{X}, d, \varepsilon)=\sup \left\{s \geq 0 \mid \mathcal{H}_{\varepsilon}^{s}(\mathcal{X}, d) \geq 1\right\} .
$$

\footnotetext{
${ }^{3}$ We do not use mean topological dimension in the paper. So we skip to define it.
} 
The Hausdorff dimension of $(\mathcal{X}, d)$ is given by

$$
\operatorname{dim}_{\mathrm{H}}(\mathcal{X}, d)=\lim _{\varepsilon \rightarrow 0} \operatorname{dim}_{\mathrm{H}}(\mathcal{X}, d, \varepsilon)
$$

Given a homeomorphism $T: \mathcal{X} \rightarrow \mathcal{X}$, we define metrics $d_{N}^{T}(N \geq 1)$ on $\mathcal{X}$ by

$$
d_{N}^{T}(x, y)=\max _{0 \leq n<N} d\left(T^{n} x, T^{n} y\right)
$$

We define the entropy at the resolution $\varepsilon>0$ by

$$
S(\mathcal{X}, T, d, \varepsilon)=\lim _{N \rightarrow \infty} \frac{\log \#\left(\mathcal{X}, d_{N}^{T}, \varepsilon\right)}{N} .
$$

This limit exists because $\log \#\left(\mathcal{X}, d_{N}^{T}, \varepsilon\right)$ is suadditive in $N$. We define the upper and lower metric mean dimensions by

$$
\overline{\operatorname{mdim}}_{\mathrm{M}}(\mathcal{X}, T, d)=\limsup _{\varepsilon \rightarrow 0} \frac{S(\mathcal{X}, T, d, \varepsilon)}{\log (1 / \varepsilon)}, \quad \underline{\operatorname{mdim}}_{\mathrm{M}}(\mathcal{X}, T, d)=\liminf _{\varepsilon \rightarrow 0} \frac{S(\mathcal{X}, T, d, \varepsilon)}{\log (1 / \varepsilon)} .
$$

When the upper and lower limits coincide, we denote the common value by $\operatorname{mim}_{\mathrm{M}}(\mathcal{X}, T, d)$.

We define the upper and lower mean Hausdorff dimensions by

$$
\begin{aligned}
& \overline{\operatorname{mdim}}_{\mathrm{H}}(\mathcal{X}, T, d)=\lim _{\varepsilon \rightarrow 0}\left(\limsup _{N \rightarrow \infty} \frac{\operatorname{dim}_{\mathrm{H}}\left(\mathcal{X}, d_{N}, \varepsilon\right)}{N}\right), \\
& \underline{\operatorname{mim}}_{\mathrm{H}}(\mathcal{X}, T, d)=\lim _{\varepsilon \rightarrow 0}\left(\liminf _{N \rightarrow \infty} \frac{\operatorname{dim}_{\mathrm{H}}\left(\mathcal{X}, d_{N}, \varepsilon\right)}{N}\right) .
\end{aligned}
$$

When these two quantities are equal to each other, we denote the common value by $\operatorname{mim}_{\mathrm{H}}(\mathcal{X}, T, d)$.

The following is the dynamical analogue of the fact that Minkowski dimension bounds Hausdorff dimension. It was proved in [LT19, Proposition 3.2].

\section{Proposition 2.1.}

$$
\underline{\operatorname{mim}}_{\mathrm{H}}(\mathcal{X}, T, d) \leq \overline{\operatorname{mdim}}_{\mathrm{H}}(\mathcal{X}, T, d) \leq \underline{\operatorname{mdim}}_{\mathrm{M}}(\mathcal{X}, T, d) \leq \overline{\operatorname{mdim}}_{\mathrm{M}}(\mathcal{X}, T, d)
$$

Remark 2.2. Here is one remark about the notation. In the paper [LT19], the lower mean Hausdorff dimension played no role. So the upper mean Hausdorff dimension was simply denoted by $\operatorname{mim}_{\mathrm{H}}(\mathcal{X}, T, d)$ in [LT19].

2.2. Mutual information. Let $(\Omega, \mathbb{P})$ be a probability space. Let $\mathcal{X}$ and $\mathcal{Y}$ be measurable spaces, and let $X: \Omega \rightarrow \mathcal{X}$ and $Y: \Omega \rightarrow \mathcal{Y}$ be measurable maps. We want to define their mutual information $I(X ; Y)$ as the measure of the amount of information $X$ and $Y$ share. (This will be used in the definition of rate distortion function in the next subsection.) The basic reference is [CT06]. 
Case 1: When $\mathcal{X}$ and $\mathcal{Y}$ are finite sets. In this case ${ }^{4}$ we set

$$
\begin{aligned}
I(X ; Y) & =H(X)+H(Y)-H(X, Y)=H(X)-H(Y \mid X) \\
& =\sum_{x \in \mathcal{X}, y \in \mathcal{Y}} \mathbb{P}(X=x, Y=y) \log \frac{\mathbb{P}(X=x, Y=y)}{\mathbb{P}(X=x) \mathbb{P}(Y=y)} .
\end{aligned}
$$

Here we have used the convention that $0 \log (0 / a)=0$ for all $a \geq 0$.

Case 2: General case. Let $f: \mathcal{X} \rightarrow A$ and $g: \mathcal{Y} \rightarrow B$ be measurable maps such that $A$ and $B$ are finite sets. Then we can define $I(f \circ X ; g \circ Y)$ by $(2.1)$. We define $I(X ; Y)$ as the supremum of $I(f \circ X ; g \circ Y)$ over all finite range measurable maps $f$ on $\mathcal{X}$ and $g$ on $\mathcal{Y}$. When $\mathcal{X}$ and $\mathcal{Y}$ are finite sets, this definition is compatible with (2.1). (Namely the supremum is attained when $f$ and $g$ are the identity maps.)

The mutual information is symmetric and nonnegative: $I(X ; Y)=I(Y ; X) \geq 0$. The following basic result immediately follows from the above definition.

Lemma 2.3 (Data-Processing inequality). Let $\mathcal{Z}$ and $\mathcal{W}$ be measurable spaces. If $f$ : $\mathcal{X} \rightarrow \mathcal{Z}$ and $g: \mathcal{Y} \rightarrow \mathcal{W}$ be measurable maps, then $I(f(X) ; g(Y)) \leq I(X ; Y)$.

2.3. Rate distortion theory. Here we introduce rate distortion function. As Shannon entropy is the fundamental limit of lossless data compression, rate distortion function is the fundamental limit of lossy data compression ${ }^{5}$. A friendly introduction can be found in [CT06, Chapter 10].

Let $(\mathcal{X}, T)$ be a dynamical system with a metric $d$ and an invariant Borel probability measure $\mu$. We define the rate distortion function $R(d, \mu, \varepsilon)(\varepsilon>0)$ as the infimum of

$$
\frac{I(X ; Y)}{N},
$$

where $N$ runs over natural numbers, $X$ and $Y=\left(Y_{0}, \ldots, Y_{N-1}\right)$ are random variables defined on some probability space $(\Omega, \mathbb{P})$ such that

- $X$ takes values in $\mathcal{X}$ according to the law $\mu$.

- $Y_{0}, \ldots, Y_{N-1}$ take values in $\mathcal{X}$ and satisfy

$$
\mathbb{E}\left(\frac{1}{N} \sum_{n=0}^{N-1} d\left(T^{n} X, Y_{n}\right)\right)<\varepsilon .
$$

The condition (2.2) means that $Y=\left(Y_{0}, \ldots, Y_{N-1}\right)$ approximates the stochastic process $X, T X, \ldots, T^{N-1} X$ within the averaged distortion bound by $\varepsilon$. We define the upper and lower rate distortion dimensions $\overline{\operatorname{rdim}}(\mathcal{X}, T, d, \mu)$ and $\underline{\operatorname{rdim}}(\mathcal{X}, T, d, \mu)$ by (1.2) in $\S 1.2$.

The rate distortion function $R(d, \mu, \varepsilon)$ is the minimum rate when we try to quantize the process $\left\{T^{n} X\right\}_{n \in \mathbb{Z}}$ within the averaged distortion bound by $\varepsilon$. See [CT06, Chapter 10], [Gra90, Chapter 11] and [ECG94, LDN79] for the precise meaning of this statement.

\footnotetext{
${ }^{4}$ We always assume that the $\sigma$-algebras of finite sets are the largest ones, i.e. the sets of all subsets.

${ }^{5}$ For example, expanding a given signal in a wavelet basis and discarding a small terms.
} 
The rest of this subsection is not used in the proof of Theorem 1.1. We include this for providing readers a wider view of the subject. A metric $d$ is said to have the tame growth of covering numbers if for any $\delta>0$

$$
\lim _{\varepsilon \rightarrow 0} \varepsilon^{\delta} \log \#(\mathcal{X}, d, \varepsilon)=0 .
$$

Note that this is purely a condition on the metric structure and does not involve dynamics. (2.3) is a mild condition. It is known ([LT19, Lemma 3.10]) that every compact metrizable space admits a metric satisfying (2.3). For example, the metrics (1.3) and (1.6) on the shift space $A^{\mathbb{Z}^{2}}$ satisfy (2.3). The following theorem [LT19, Proposition 3.2, Theorem 3.11] provides a link between rate distortion dimension and various mean dimensions. Here we denote by $\mathscr{M}^{T}(\mathcal{X})$ the set of all invariant Borel probability measures on $\mathcal{X}$.

\section{Theorem 2.4.}

$$
\overline{\operatorname{rdim}}(\mathcal{X}, T, d, \mu) \leq \overline{\operatorname{mdim}}_{\mathrm{M}}(\mathcal{X}, T, d), \quad \underline{\operatorname{rdim}}(\mathcal{X}, T, d, \mu) \leq \underline{\operatorname{mdim}}_{\mathrm{M}}(\mathcal{X}, T, d) .
$$

If $d$ has the tame growth of covering numbers then

$$
\overline{\operatorname{mdim}}_{\mathrm{H}}(\mathcal{X}, T, d) \leq \sup _{\mu \in \mathscr{M}^{T}(\mathcal{X})} \underline{\operatorname{rdim}}(\mathcal{X}, T, d, \mu)
$$

\section{Proof of Theorem 1.1}

First we recall the notations of $\S 1.3 . A^{\mathbb{Z}^{2}}$ is the $\mathbb{Z}^{2}$-full shift on the alphabet (finite set) $A$ with the shifts $\sigma_{1}$ and $\sigma_{2}$. Fix $\alpha>1$ and we define the metric $d$ on $A^{\mathbb{Z}^{2}}$ by

$$
d(x, y)=\alpha^{-\min \left\{|u|_{\infty} \mid x_{u} \neq y_{u}\right\}} .
$$

Let $\mathcal{X} \subset A^{\mathbb{Z}^{2}}$ be a subshift (closed shift-invariant set) with a Borel probability measure $\mu$ invariant under both $\sigma_{1}$ and $\sigma_{2}$.

The proof of Theorem 1.1 is divided into 4 steps:

(1) Prove the upper bound on the upper metric mean dimension

$$
\overline{\operatorname{mdim}}_{\mathrm{M}}\left(\mathcal{X}, \sigma_{1}, d\right) \leq \frac{2 h_{\mathrm{top}}\left(\mathcal{X}, \sigma_{1}, \sigma_{2}\right)}{\log \alpha}
$$

(2) Prove the lower bound on the lower mean Hausdorff dimension

$$
\underline{\operatorname{mim}}_{\mathrm{H}}\left(\mathcal{X}, \sigma_{1}, d\right) \geq \frac{2 h_{\mathrm{top}}\left(\mathcal{X}, \sigma_{1}, \sigma_{2}\right)}{\log \alpha} .
$$

(3) Prove the upper bound on the upper rate distortion dimension

$$
\overline{\operatorname{rdim}}\left(\mathcal{X}, \sigma_{1}, d, \mu\right) \leq \frac{2 h_{\mu}\left(\mathcal{X}, \sigma_{1}, \sigma_{2}\right)}{\log \alpha} .
$$

(4) Prove the lower bound on the lower rate distortion dimension

$$
\underline{\operatorname{rdim}}\left(\mathcal{X}, \sigma_{1}, d, \mu\right) \geq \frac{2 h_{\mu}\left(\mathcal{X}, \sigma_{1}, \sigma_{2}\right)}{\log \alpha} .
$$


Since we know $\underline{\operatorname{mdim}}_{\mathrm{H}}\left(\mathcal{X}, \sigma_{1}, d\right) \leq \overline{\operatorname{mdim}}_{\mathrm{M}}\left(\mathcal{X}, \sigma_{1}, d\right)$ by Proposition 2.1 , the steps $(1)$ and (2) show

$$
\operatorname{mdim}_{\mathrm{H}}\left(\mathcal{X}, \sigma_{1}, d\right)=\operatorname{mdim}_{\mathrm{M}}\left(\mathcal{X}, \sigma_{1}, d\right)=\frac{2 h_{\mathrm{top}}\left(\mathcal{X}, \sigma_{1}, \sigma_{2}\right)}{\log \alpha}
$$

The steps (3) and (4) show

$$
\operatorname{rdim}\left(\mathcal{X}, \sigma_{1}, d, \mu\right)=\frac{2 h_{\mu}\left(\mathcal{X}, \sigma_{1}, \sigma_{2}\right)}{\log \alpha}
$$

The steps (1) and (3) are easy. The step (2) is the most involved. The four steps are independent of each other.

For $\Omega \subset \mathbb{Z}^{2}$ we denote by $\pi_{\Omega}: \mathcal{X} \rightarrow A^{\Omega}$ the natural projection. As in $\S 2.1$ we set $d_{N}^{\sigma_{1}}(x, y)=\max _{0 \leq n<N} d\left(\sigma_{1}^{n} x, \sigma_{1}^{n} y\right)$ for $N>0$. In this section, intervals mean discrete intervals. Namely, for example, $[a, b]=\{a, a+1, \ldots, b-1, b\}$ and $(a, b)=\{a+1, a+$ $2, \ldots, b-1\}$ for integers $a \leq b$.

3.1. Step 1: Proof of $\overline{\operatorname{mdim}}_{\mathrm{M}}\left(\mathcal{X}, \sigma_{1}, d\right) \leq 2 h_{\text {top }}\left(\mathcal{X}, \sigma_{1}, \sigma_{2}\right) / \log \alpha$. Let $0<\varepsilon<1$ and take a natural number $M$ with $\alpha^{-M}<\varepsilon \leq \alpha^{-M+1}$. Then

$$
\#\left(\mathcal{X}, d_{N}^{\sigma_{1}}, \varepsilon\right) \leq\left|\pi_{(-M, N+M) \times(-M, M)}(\mathcal{X})\right|
$$

(Here $|\cdot|$ denotes the cardinality.) Since $(M-1) \log \alpha \leq \log (1 / \varepsilon)<M \log \alpha$,

$$
\begin{aligned}
\overline{\operatorname{mdim}}_{\mathrm{M}}\left(\mathcal{X}, \sigma_{1}, d\right) & =\limsup _{\varepsilon \rightarrow 0}\left(\lim _{N \rightarrow \infty} \frac{\log \#\left(\mathcal{X}, d_{N}^{\sigma_{1}}, \varepsilon\right)}{N \log (1 / \varepsilon)}\right) \\
& \leq \lim _{M \rightarrow \infty}\left(\lim _{N \rightarrow \infty} \frac{\log \left|\pi_{(-M, N+M) \times(-M, M)}(\mathcal{X})\right|}{N(M-1) \log \alpha}\right) \\
& =\frac{2 h_{\text {top }}\left(\mathcal{X}, \sigma_{1}, \sigma_{2}\right)}{\log \alpha}
\end{aligned}
$$

3.2. Step 2: Proof of $\underline{\operatorname{mdim}}_{\mathrm{H}}\left(\mathcal{X}, \sigma_{1}, d\right) \geq 2 h_{\mathrm{top}}\left(\mathcal{X}, \sigma_{1}, \sigma_{2}\right) / \log \alpha$. First we prepare some terminologies about the geometry of $\mathbb{Z}^{2}$. In this subsection rectangles mean sets of the form $[a, b] \times[c, d]$ in $\mathbb{Z}^{2}$ for integers $a \leq b$ and $c \leq d$. For a rectangle $R=[a, b] \times[c, d]$ we define a new rectangle $3 R$ by

$$
3 R=[2 a-b, 2 b-a] \times[2 c-d, 2 d-c] .
$$

We have $|3 R|=(3 b-3 a+1)(3 d-3 c+1) \leq 9|R|$.

For two rectangles $R=[a, b] \times[c, d]$ and $R^{\prime}=\left[a^{\prime}, b^{\prime}\right] \times\left[c^{\prime}, d^{\prime}\right]$, we denote by $R \leq R^{\prime}$ if $b-a \leq b^{\prime}-a^{\prime}$ and $d-c \leq d^{\prime}-c^{\prime}$. This defines an order among rectangles. (Strictly speaking, this is a "pre-order" because $R \leq R^{\prime}$ and $R^{\prime} \leq R$ does not imply $R=R^{\prime}$.) A set of rectangles $\left\{R_{1}, \ldots, R_{n}\right\}$ is said to be totally ordered if any two elements are comparable, i.e. for any $R_{i}$ and $R_{j}$ we have either $R_{i} \leq R_{j}$ or $R_{j} \leq R_{i}$. 
The following trivial fact will be used later: Suppose $\left\{R_{1}, \ldots, R_{n}\right\}$ is totally ordered. If a set of rectangles $\left\{R_{1}^{\prime}, \ldots, R_{n^{\prime}}^{\prime}\right\}$ has the property that each $R_{i}^{\prime}$ is a parallel translation of some $R_{j}$ (namely $R_{i}^{\prime}=u+R_{j}$ for some $u \in \mathbb{Z}^{2}$ ) then $\left\{R_{1}^{\prime}, \ldots, R_{n^{\prime}}^{\prime}\right\}$ is also totally ordered.

The next lemma is a kind of finite Vitali covering lemma ([EW11, Lemma 2.27]) adapted to our situation.

Lemma 3.1. Suppose a set of rectangles $\left\{R_{1}, \ldots, R_{n}\right\}$ is totally ordered. Then we can find a disjoint subfamily $\left\{R_{i_{1}}, \ldots, R_{i_{m}}\right\}$ satisfying

$$
R_{1} \cup \cdots \cup R_{n} \subset 3 R_{i_{1}} \cup 3 R_{i_{2}} \cup \cdots \cup 3 R_{i_{m}} .
$$

Note that this implies

$$
\left|R_{i_{1}} \cup \cdots \cup R_{i_{m}}\right| \geq \frac{1}{9}\left|R_{1} \cup \cdots \cup R_{n}\right| \text {. }
$$

Proof. We use a simple greedy algorithm. We first choose (one of) the largest rectangle, say $R_{i_{1}}$. Next, suppose we have chosen $R_{i_{1}}, \ldots, R_{i_{k}}$. We choose as $R_{i_{k+1}}$ the largest rectangle disjoint to $R_{i_{1}} \cup \cdots \cup R_{i_{k}}$. If there is no such a rectangle, the algorithm stops.

Suppose the algorithm stops after $m$ steps. For any $R_{j}$ there exists $R_{i_{k}}$ with $R_{i_{k}} \geq R_{j}$ and $R_{i_{k}} \cap R_{j} \neq \emptyset$. This implies $R_{j} \subset 3 R_{i_{k}}$.

For two sets $\Omega, \Lambda \subset \mathbb{Z}^{2}$ we define $\partial_{\Lambda} \Omega$ as the set of $u \in \mathbb{Z}^{2}$ such that $u+\Lambda$ has non-empty intersections both with $\Omega$ and $\mathbb{Z}^{2} \backslash \Omega$. We set $\operatorname{Int}_{\Lambda} \Omega=\Omega \backslash \partial_{\Lambda} \Omega$. This is the set of $u \in \Omega$ with $u+\Lambda \subset \Omega$.

Let $R \subset \mathbb{Z}^{2}$ be a rectangle. A subset $C \subset \mathcal{X}$ is called a cylinder over $R$ if there is $x \in \mathcal{X}$ such that $C$ is equal to the set of $y \in \mathcal{X}$ satisfying $\pi_{R}(y)=\pi_{R}(x)$.

Set

$$
s=\frac{2 h_{\mathrm{top}}\left(\mathcal{X}, \sigma_{1}, \sigma_{2}\right)}{\log \alpha} .
$$

Suppose $\underline{\operatorname{mim}}_{\mathrm{H}}\left(\mathcal{X}, \sigma_{1}, d\right)<s$. We would like to get a contradiction. We fix $\varepsilon>0$ satisfying $\underline{\operatorname{mim}}_{\mathrm{H}}\left(\mathcal{X}, \sigma_{1}, d\right)<s-2 \varepsilon$.

Lemma 3.2. For any finite subset $\Lambda \subset \mathbb{Z}^{2}$ and any positive number $L$, we can find rectangles $R_{1}, \ldots, R_{M} \subset \mathbb{Z}^{2}$ and subsets $C_{1}, \ldots, C_{M} \subset \mathcal{X}$ such that

- Each $C_{m}$ is a cylinder over $R_{m}$ and they satisfy $\mathcal{X}=\bigcup_{m=1}^{M} C_{m}$.

- All the rectangles $R_{m}$ contain the origin, and they are all sufficiently large so that

$$
\left|\partial_{\Lambda} R_{m}\right|<\frac{\left|R_{m}\right|}{L}, \quad\left|R_{m}\right|>L
$$

- The rectangles $R_{1}, \ldots, R_{M}$ are totally ordered and satisfy

$$
\sum_{m=1}^{M} \alpha^{-\frac{1}{2}(s-\varepsilon)\left|R_{m}\right|}<1 .
$$

Proof. We choose a natural number $r_{0}$ such that 
- Every $r \geq r_{0}$ satisfies $(s-2 \varepsilon) r<(s-\varepsilon)(r-1)$.

- If a rectangle $R=[a, b] \times[c, d] \subset \mathbb{Z}^{2}$ satisfies $b-a \geq r_{0}$ and $d-c \geq r_{0}$ then

$$
\left|\partial_{\Lambda} R\right|<\frac{|R|}{L}, \quad|R|>L .
$$

From $\underline{\operatorname{mdim}}_{\mathrm{H}}\left(\mathcal{X}, \sigma_{1}, d\right)<s-2 \varepsilon$, we can find $N>0$ satisfying

$$
\frac{1}{N} \operatorname{dim}_{\mathrm{H}}\left(\mathcal{X}, d_{N}^{\sigma_{1}}, \alpha^{-r_{0}}\right)<s-2 \varepsilon .
$$

This implies that there exists a covering $\mathcal{X}=E_{1} \cup \cdots \cup E_{M}$ satisfying

$$
\operatorname{diam}\left(E_{m}, d_{N}^{\sigma_{1}}\right)<\alpha^{-r_{0}}(\forall 1 \leq m \leq M), \quad \sum_{m=1}^{M}\left(\operatorname{diam}\left(E_{m}, d_{N}^{\sigma_{1}}\right)\right)^{(s-2 \varepsilon) N}<1 .
$$

Set $\alpha^{-r_{m}}:=\operatorname{diam}\left(E_{m}, d_{N}^{\sigma_{1}}\right)$. Then $r_{m}$ is a natural number with $r_{m}>r_{0}$. Choose a point $x_{m}$ from each $E_{m}$, and let $C_{m} \subset \mathcal{X}$ be a cylinder over the rectangle

$$
R_{m}:=\left[-r_{m}+1, N+r_{m}-2\right] \times\left[-r_{m}+1, r_{m}-1\right]
$$

defined by $C_{m}=\pi_{R_{m}}^{-1}\left(\pi_{R_{m}}\left(x_{m}\right)\right)$. Then $E_{m} \subset C_{m}$ and hence $\mathcal{X}=C_{1} \cup \cdots \cup C_{m}$. The rectangles $R_{m}$ are totally ordered $\left(R_{m} \leq R_{m^{\prime}}\right.$ if and only if $\left.r_{m} \leq r_{m^{\prime}}\right)$.

Recall that $r_{m}>r_{0}$ for all $1 \leq m \leq M$. From the choice of $r_{0}$,

$$
\left|\partial_{\Lambda} R_{m}\right|<\frac{\left|R_{m}\right|}{L}, \quad\left|R_{m}\right|>L .
$$

From $\left|R_{m}\right|=\left(N+2 r_{m}-2\right)\left(2 r_{m}-1\right) \geq N\left(2 r_{m}-1\right)$,

$$
\begin{aligned}
\frac{1}{2}(s-\varepsilon)\left|R_{m}\right| & \geq \frac{1}{2}(s-\varepsilon) N\left(2 r_{m}-1\right) \\
& >\frac{1}{2}(s-2 \varepsilon) N\left(2 r_{m}\right) \quad \text { by the choice of } r_{0} \\
& =(s-2 \varepsilon) N r_{m} .
\end{aligned}
$$

Hence

$$
\alpha^{-\frac{1}{2}(s-\varepsilon)\left|R_{m}\right|}<\alpha^{-(s-2 \varepsilon) N r_{m}}=\left(\operatorname{diam}\left(E_{m}, d_{N}^{\sigma_{1}}\right)\right)^{(s-2 \varepsilon) N} .
$$

Therefore

$$
\sum_{m=1}^{M} \alpha^{-\frac{1}{2}(s-\varepsilon)\left|R_{m}\right|}<\sum_{m=1}^{M}\left(\operatorname{diam}\left(E_{m}, d_{N}^{\sigma_{1}}\right)\right)^{(s-2 \varepsilon) N}<1
$$

We choose a real number $0<\delta<1 / 2$ and a natural number $p$ satisfying the following conditions.

$$
\left(\frac{17}{18}\right)^{p}<\delta, \quad H(\delta)+\delta \log p<\frac{\varepsilon}{8} \log \alpha, \quad|A|^{\delta}<\alpha^{\varepsilon / 8} .
$$

Here $H(\delta)=-\delta \log \delta-(1-\delta) \log (1-\delta)$. (Recall that the base of the logarithm is two.) The first condition is satisfied for $p \approx \log (1 / \delta)$. Then we choose a sufficiently small $\delta$ satisfying the second and third conditions. 
By using Lemma 3.2 iteratively, we find rectangles $R_{i, m}$ and subsets $C_{i, m} \subset \mathcal{X}$ for $i=1, \ldots, p$ and $m=1, \ldots, M_{i}$ (where $M_{i}$ is a natural number depending on $i$ ) satisfying the following conditions.

(a) Each $C_{i, m}$ is a cylinder over $R_{i, m}$. For each $1 \leq i \leq p$ we have $\mathcal{X}=\bigcup_{m=1}^{M_{i}} C_{i, m}$.

(b) For each $1 \leq i \leq p$, the rectangles $R_{i, 1}, R_{i, 2}, \ldots, R_{i, M_{i}}$ are totally ordered and satisfy

$$
\sum_{m=1}^{M_{i}} \alpha^{-\frac{1}{2}(s-\varepsilon)\left|R_{i, m}\right|}<1 .
$$

(c) All the rectangles $R_{i, m}$ contain the origin and they satisfy $\left|R_{i, m}\right|>1 / \delta$.

(d) Set $\hat{R}_{i}=\bigcup_{m=1}^{M_{i}} R_{i, m}$. Then for all $j<i$ and $m=1, \ldots, M_{j}$ we have

$$
\left|\partial_{\hat{R}_{i}} R_{j, m}\right|<\frac{\delta}{4}\left|R_{j, m}\right|
$$

Roughly speaking, the condition (d) means that the rectangles in one level (say, $j$ ) are much larger than the rectangles in higher levels (say, $i>j$ ). The construction goes from the level $p$ to the bottom. First, by Lemma 3.2, we construct $R_{p, m}$ and $C_{p, m}$. Next, by using the lemma again, we construct $R_{p-1, m}$ and $C_{p-1, m}$. We continue this process until we come to the first level $\left(R_{1, m}\right.$ and $\left.C_{1, m}\right)$. The condition $(\mathrm{d})$ connects the constructions in different levels.

Lemma 3.3. If $N>0$ is sufficiently large then the following statement holds. For each $x \in \mathcal{X}$ we can choose a subset

$$
D(x) \subset\left\{(u, i, m) \mid u \in[0, N-1]^{2}, 1 \leq i \leq p, 1 \leq m \leq M_{i}\right\}
$$

such that

(1) For $(u, i, m) \in D(x)$, we have $\sigma^{u}(x) \in C_{i, m}$ and $u+R_{i, m} \subset[0, N-1]^{2}$.

(2) If $(u, i, m)$ and $\left(u^{\prime}, i^{\prime}, m^{\prime}\right)$ are two different elements of $D(x)$, then $\left(u+R_{i, m}\right) \cap$ $\left(u^{\prime}+R_{i^{\prime}, m^{\prime}}\right)=\emptyset$. In particular (recall that $R_{i, m}$ contain the origin), $u \neq u^{\prime}$.

(3) We have

$$
\left|[0, N-1]^{2} \backslash \bigcup_{(u, i, m) \in D(x)}\left(u+R_{i, m}\right)\right|<\delta N^{2} .
$$

Proof. Let $N$ be sufficiently large so that

$$
\left|\partial_{\hat{R}_{i}}[0, N-1]^{2}\right|<\frac{\delta}{4} N^{2} \text { for all } 1 \leq i \leq p .
$$

Here recall that $\hat{R}_{i}=\bigcup_{m=1}^{M_{i}} R_{i, m}$. Fix $x \in \mathcal{X}$. Set $E_{0}=[0, N-1]^{2}$. We will inductively construct $E_{0} \supset E_{1} \supset E_{2} \supset \cdots \supset E_{p}$.

Suppose we have defined $E_{0}, E_{1}, \ldots, E_{i-1}$. Consider the following set of rectangles:

$$
\left\{u+R_{i, m} \mid u \in E_{i-1}, m \in\left[1, M_{i}\right] \text { with } \sigma^{u}(x) \in C_{i, m} \text { and } u+R_{i, m} \subset E_{i-1}\right\} \text {. }
$$


Since $R_{i, 1}, \ldots, R_{i, M_{i}}$ are totally ordered, so is (3.4). (Here the point is that $i$ is fixed.) The rectangles (3.4) cover $\operatorname{Int}_{\hat{R}_{i}} E_{i-1}$. Then by Lemma 3.1, we can find a subset

$$
D_{i}(x) \subset\left\{(u, m) \mid u \in E_{i-1}, 1 \leq m \leq M_{i}\right\}
$$

such that

- For $(u, m) \in D_{i}(x)$, we have $\sigma^{u}(x) \in C_{i, m}$ and $u+R_{i, m} \subset E_{i-1}$.

- If $(u, m)$ and $\left(u^{\prime}, m^{\prime}\right)$ are two different elements of $D_{i}(x)$ then $\left(u+R_{i, m}\right) \cap\left(u^{\prime}+\right.$ $\left.R_{i, m^{\prime}}\right)=\emptyset$.

- The rectangles $u+R_{i, m},(u, m) \in D_{i}(x)$, cover at least one-ninth of $\operatorname{Int}_{\hat{R}_{i}} E_{i-1}$ :

$$
\left|\bigcup_{(u, m) \in D_{i}(x)}\left(u+R_{i, m}\right)\right| \geq \frac{1}{9}\left|\operatorname{Int}_{\hat{R}_{i}} E_{i-1}\right| .
$$

We set

$$
E_{i}=E_{i-1} \backslash \bigcup_{(u, m) \in D_{i}(x)}\left(u+R_{i, m}\right)
$$

We define $D(x)$ by

$$
D(x)=\left\{(u, i, m) \mid 1 \leq i \leq p,(u, m) \in D_{i}(x)\right\} .
$$

The properties (1) and (2) of $D(x)$ immediately follow from the construction. The property (3) is equivalent to the claim that $\left|E_{p}\right|<\delta N^{2}$. We will prove this.

Suppose $\left|E_{p}\right| \geq \delta N^{2}$. Then we also have $\left|E_{i-1}\right| \geq \delta N^{2}$ for all $1 \leq i \leq p$. We estimate $\left|\partial_{\hat{R}_{i}} E_{i-1}\right|$ for $1 \leq i \leq p$. We have

$$
\partial_{\hat{R}_{i}} E_{i-1} \subset \partial_{\hat{R}_{i}}[0, N-1]^{2} \cup \bigcup_{j=1}^{i-1} \bigcup_{(u, m) \in D_{j}(x)} \partial_{\hat{R}_{i}}\left(u+R_{j, m}\right) .
$$

Recall (3.3) and $\left|\partial_{\hat{R}_{i}} R_{j, m}\right|<(\delta / 4)\left|R_{j, m}\right|$ for $j<i$ by the condition (d) of the choice of $R_{i, m}$. Then

$$
\begin{aligned}
\left|\partial_{\hat{R}_{i}} E_{i-1}\right| & \leq\left|\partial_{\hat{R}_{i}}[0, N-1]^{2}\right|+\sum_{j=1}^{i-1} \sum_{(u, m) \in D_{j}(x)}\left|\partial_{\hat{R}_{i}}\left(u+R_{j, m}\right)\right| \\
& <\frac{\delta}{4} N^{2}+\frac{\delta}{4} \sum_{j=1}^{i-1} \sum_{(u, m) \in D_{j}(x)}\left|u+R_{j, m}\right| .
\end{aligned}
$$

The rectangles $u+R_{j, m}, 1 \leq j \leq i-1$ and $(u, m) \in D_{j}(x)$, are disjoint and contained in $[0, N-1]^{2}$. Therefore

$$
\sum_{j=1}^{i-1} \sum_{(u, m) \in D_{j}(x)}\left|u+R_{j, m}\right| \leq N^{2}
$$


Thus $\left|\partial_{\hat{R}_{i}} E_{i-1}\right|<(\delta / 2) N^{2}$. Since we assumed $\left|E_{i-1}\right| \geq \delta N^{2}$, we have $\left|\partial_{\hat{R}_{i}} E_{i-1}\right|<$ $(1 / 2)\left|E_{i-1}\right|$. Namely

$$
\left|\operatorname{Int}_{\hat{R}_{i}} E_{i-1}\right|>\frac{1}{2}\left|E_{i-1}\right|
$$

From (3.5),

$$
\left|\bigcup_{(u, m) \in D_{i}(x)}\left(u+R_{i, m}\right)\right| \geq \frac{1}{9}\left|\operatorname{Int}_{\hat{R}_{i}} E_{i-1}\right|>\frac{1}{18}\left|E_{i-1}\right| .
$$

So we get

$$
\left|E_{i}\right|=\left|E_{i-1} \backslash \bigcup_{(u, m) \in D_{i}(x)}\left(u+R_{i, m}\right)\right|<\frac{17}{18}\left|E_{i-1}\right| .
$$

This holds for all $1 \leq i \leq p$. Therefore

$$
\left|E_{p}\right|<\left(\frac{17}{18}\right)^{p}\left|E_{0}\right|=\left(\frac{17}{18}\right)^{p} N^{2}
$$

Recall that $p$ satisfies $(17 / 18)^{p}<\delta$ by (3.1). So $\left|E_{p}\right|<\delta N^{2}$. This is a contradiction.

In the rest of this subsection, $N$ is assumed to be so large that the statement of Lemma 3.3 holds. For each $x \in \mathcal{X}$ we define $\underline{D}(x) \subset[0, N-1]^{2} \times[1, p]$ as the set of $(u, i) \in$ $[0, N-1]^{2} \times[1, p]$ such that there exists $m \in\left[1, M_{i}\right]$ with $(u, i, m) \in D(x)$. (Notice that the sets $D(x)$ and $\underline{D}(x)$ depend on $N$. So it might be better to use the notations $D^{(N)}(x)$ and $\underline{D}^{(N)}(x)$. But we prefer the simpler ones here.)

Lemma 3.4. If $N$ is sufficiently large then the number of possibilities of $\underline{D}(x)$ is bounded as follows:

$$
|\{\underline{D}(x) \mid x \in \mathcal{X}\}|<\alpha^{(\varepsilon / 8) N^{2}} .
$$

Proof. We use the well-known bound on the binomial coefficient:

$$
\left(\begin{array}{l}
n \\
k
\end{array}\right) \leq 2^{n H(k / n)}
$$

This follows from

$$
1=\left\{\frac{k}{n}+\left(1-\frac{k}{n}\right)\right\}^{n} \geq\left(\begin{array}{l}
n \\
k
\end{array}\right)\left(\frac{k}{n}\right)^{k}\left(1-\frac{k}{n}\right)^{n-k}=\left(\begin{array}{l}
n \\
k
\end{array}\right) 2^{-n H(k / n)} .
$$

Let $x \in \mathcal{X}$ and set $D(x)=\left\{\left(u_{1}, i_{1}, m_{1}\right), \ldots,\left(u_{k}, i_{k}, m_{k}\right)\right\}$. (Then we have $\underline{D}(x)=$ $\left.\left\{\left(u_{1}, i_{1}\right), \ldots,\left(u_{k}, i_{k}\right)\right\}.\right)$ By $(1)$ and $(2)$ of Lemma $3.3, u_{1}, \ldots, u_{k}$ are different from each other, and the rectangles $u_{1}+R_{i_{1}, m_{1}}, \ldots, u_{k}+R_{i_{k}, m_{k}}$ are disjoint and contained in $[0, N-$ $1]^{2}$. Since $\left|R_{i, m}\right|>1 / \delta$ (the condition (c) of the choice of $R_{i, m}$ ), we have $k<\delta N^{2}$. 
Then the number of possibilities of $\underline{D}(x)$ is bounded by

$$
\begin{aligned}
& \underbrace{\left\{\left(\begin{array}{c}
N^{2} \\
1
\end{array}\right)+\left(\begin{array}{c}
N^{2} \\
2
\end{array}\right)+\cdots+\left(\begin{array}{c}
N^{2} \\
\left\lfloor N^{2}\right\rfloor
\end{array}\right)\right\}}_{\text {choices of } u_{1}, \ldots, u_{k}} \times \underbrace{p^{\delta N^{2}}}_{\text {choices of } i_{1}, \ldots, i_{k}} \\
& \leq N^{2} \cdot 2^{N^{2} H(\delta)} \times p^{\delta N^{2}} \quad \text { by }(3.6) \\
& =N^{2} \cdot 2^{N^{2}(H(\delta)+\delta \log p)} .
\end{aligned}
$$

We assumed $H(\delta)+\delta \log p<(\varepsilon / 8) \log \alpha$ in (3.1). Hence, if $N$ is sufficiently large then

$$
N^{2} \cdot 2^{N^{2}(H(\delta)+\delta \log p)}<2^{N^{2}(\varepsilon / 8) \log \alpha}=\alpha^{(\varepsilon / 8) N^{2}} .
$$

Take a subset $E \subset[0, N-1]^{2} \times[1, p]$ such that there exists $x \in \mathcal{X}$ with $\underline{D}(x)=E$. We denote by $\mathcal{X}_{E}$ the set of $x \in \mathcal{X}$ with $\underline{D}(x)=E$. Let $E=\left\{\left(u_{1}, i_{1}\right),\left(u_{2}, i_{2}\right), \ldots,\left(u_{k}, i_{k}\right)\right\}$.

\section{Lemma 3.5.}

$$
\begin{aligned}
& \left|\pi_{[0, N-1]^{2}}\left(\mathcal{X}_{E}\right)\right| \cdot \alpha^{-\frac{1}{2}(s-\varepsilon) N^{2}} \\
& \leq|A|^{\delta N^{2}}\left(\sum_{m=1}^{M_{i_{1}}} \alpha^{-\frac{1}{2}(s-\varepsilon)\left|R_{i_{1}, m}\right|}\right) \times \cdots \times\left(\sum_{m=1}^{M_{i_{k}}} \alpha^{-\frac{1}{2}(s-\varepsilon)\left|R_{i_{k}, m}\right|}\right) .
\end{aligned}
$$

Proof. For $\mathbf{m}=\left(m_{1}, \ldots, m_{k}\right) \in\left[1, M_{i_{1}}\right] \times \cdots \times\left[1, M_{i_{k}}\right]$, we denote by $\mathcal{X}_{E, \mathbf{m}} \subset \mathcal{X}_{E}$ the set of $x \in \mathcal{X}_{E}$ with $D(x)=\left\{\left(u_{1}, i_{1}, m_{1}\right),\left(u_{2}, i_{2}, m_{2}\right), \ldots,\left(u_{k}, i_{k}, m_{k}\right)\right\}$. We have $\sigma^{u_{j}}(x) \in C_{i_{j}, m_{j}}$ for $x \in \mathcal{X}_{E, \mathbf{m}}$. Hence, over each rectangle $u_{j}+R_{i_{j}, m_{j}}$, the value of $\pi_{u_{j}+R_{i_{j}, m_{j}}}(x)\left(x \in \mathcal{X}_{E, \mathbf{m}}\right)$ is fixed. (Namely we have $\pi_{u_{j}+R_{i_{j}, m_{j}}}(x)=\pi_{u_{j}+R_{i_{j}, m_{j}}}\left(x^{\prime}\right)$ for any two $x, x^{\prime} \in \mathcal{X}_{E, \mathbf{m}}$.) Therefore we have

$$
\left|\pi_{[0, N-1]^{2}}\left(\mathcal{X}_{E, \mathbf{m}}\right)\right| \leq|A|^{\left|[0, N-1]^{2} \backslash \bigcup_{j=1}^{k}\left(u_{j}+R_{i_{j}, m_{j}}\right)\right|}<|A|^{\delta N^{2}}
$$

Here the second inequality follows from the condition (3) of Lemma 3.3. We decompose the left-hand side of (3.7) as

$$
\begin{aligned}
\left|\pi_{[0, N-1]^{2}}\left(\mathcal{X}_{E}\right)\right| \cdot \alpha^{-\frac{1}{2}(s-\varepsilon) N^{2}} & =\sum_{\mathbf{m}}\left|\pi_{[0, N-1]^{2}}\left(\mathcal{X}_{E, \mathbf{m}}\right)\right| \cdot \alpha^{-\frac{1}{2}(s-\varepsilon) N^{2}} \\
& \leq \sum_{\mathbf{m} \text { with } \mathcal{X}_{E, \mathbf{m}} \neq \emptyset}|A|^{\delta N^{2}} \cdot \alpha^{-\frac{1}{2}(s-\varepsilon) N^{2}}
\end{aligned}
$$

Take $\mathbf{m}=\left(m_{1}, \ldots, m_{k}\right) \in\left[1, M_{i_{1}}\right] \times \cdots \times\left[1, M_{i_{k}}\right]$ with $\mathcal{X}_{E, \mathbf{m}} \neq \emptyset$. The rectangles $u_{j}+R_{i_{j}, m_{j}}$ $(1 \leq j \leq k)$ are disjoint and contained in $[0, N-1]^{2}$ by the conditions (1) and (2) of Lemma 3.3. Hence

$$
N^{2} \geq \sum_{j=1}^{k}\left|R_{i_{j}, m_{k}}\right|
$$


So

$$
\alpha^{-\frac{1}{2}(s-\varepsilon) N^{2}} \leq \prod_{j=1}^{k} \alpha^{-\frac{1}{2}(s-\varepsilon)\left|R_{i_{j}, m_{j}}\right|} .
$$

Plugging this into (3.8), we get

$$
\left|\pi_{[0, N-1]^{2}}\left(\mathcal{X}_{E}\right)\right| \cdot \alpha^{-\frac{1}{2}(s-\varepsilon) N^{2}} \leq \sum_{\mathbf{m}}|A|^{\delta N^{2}} \prod_{j=1}^{k} \alpha^{-\frac{1}{2}(s-\varepsilon)\left|R_{i_{j}, m_{j}}\right|} .
$$

The right-hand side is equal to

$$
|A|^{\delta N^{2}}\left(\sum_{m=1}^{M_{i_{1}}} \alpha^{-\frac{1}{2}(s-\varepsilon)\left|R_{i_{1}, m}\right|}\right) \times \cdots \times\left(\sum_{m=1}^{M_{i_{k}}} \alpha^{-\frac{1}{2}(s-\varepsilon)\left|R_{i_{k}, m}\right|}\right) .
$$

We continue the estimates:

$$
\begin{aligned}
\left|\pi_{[0, N-1]^{2}}\left(\mathcal{X}_{E}\right)\right| \cdot \alpha^{-\frac{1}{2}(s-\varepsilon) N^{2}} & \leq|A|^{\delta N^{2}}\left(\sum_{m=1}^{M_{i_{1}}} \alpha^{-\frac{1}{2}(s-\varepsilon)\left|R_{i_{1}, m}\right|}\right) \times \cdots \times\left(\sum_{m=1}^{M_{i_{k}}} \alpha^{-\frac{1}{2}(s-\varepsilon)\left|R_{i_{k}, m}\right|}\right) \\
& <|A|^{\delta N^{2}} \text { by }(3.2) \\
& <\alpha^{(\varepsilon / 8) N^{2}} \quad \text { since we assumed }|A|^{\delta}<\alpha^{\varepsilon / 8} \text { in }(3.1) .
\end{aligned}
$$

The number of choices of $E \subset[0, N-1]^{2} \times[1, p]$ with $\mathcal{X}_{E} \neq \emptyset$ is bounded by $\alpha^{(\varepsilon / 8) N^{2}}$ if $N$ is sufficiently large (Lemma 3.4). Then

$$
\begin{aligned}
\left|\pi_{[0, N-1]^{2}}(\mathcal{X})\right| \cdot \alpha^{-\frac{1}{2}(s-\varepsilon) N^{2}} & =\sum_{E \text { with } \mathcal{X}_{E} \neq \emptyset}\left|\pi_{[0, N-1]^{2}}\left(\mathcal{X}_{E}\right)\right| \cdot \alpha^{-\frac{1}{2}(s-\varepsilon) N^{2}} \\
& <\alpha^{(\varepsilon / 8) N^{2}} \times \alpha^{(\varepsilon / 8) N^{2}}=\alpha^{(\varepsilon / 4) N^{2}} .
\end{aligned}
$$

Therefore

$$
\left|\pi_{[0, N-1]^{2}}(\mathcal{X})\right|<\alpha^{\frac{1}{2}\left(s-\frac{\varepsilon}{2}\right) N^{2}}
$$

Namely

Letting $N \rightarrow \infty$

$$
\frac{\log \left|\pi_{[0, N-1]^{2}}(\mathcal{X})\right|}{N^{2}}<\frac{1}{2}\left(s-\frac{\varepsilon}{2}\right) \log \alpha
$$

$$
h_{\text {top }}\left(\mathcal{X}, \sigma_{1}, \sigma_{2}\right) \leq \frac{1}{2}\left(s-\frac{\varepsilon}{2}\right) \log \alpha<\frac{1}{2} s \log \alpha=h_{\text {top }}\left(\mathcal{X}, \sigma_{1}, \sigma_{2}\right) .
$$

This is a contradiction.

Remark 3.6. (1) The above proof (in particular, see the proof of Lemma 3.2) also shows a (seemingly) slightly stronger statement that

$$
\lim _{\varepsilon \rightarrow 0}\left(\inf _{N \geq 1} \frac{\operatorname{dim}_{\mathrm{H}}\left(\mathcal{X}, d_{N}^{\sigma_{1}}, \varepsilon\right)}{N}\right) \geq \frac{2 h_{\mathrm{top}}\left(\mathcal{X}, \sigma_{1}, \sigma_{2}\right)}{\log \alpha} .
$$


Combined with Step 1, the both sides actually coincide. However we do not know whether the left-hand side is an important quantity or not.

(2) The above proof (in particular, the use of covering argument) is motivated by the proof of the Shannon-McMillan-Breiman theorem (see, e.g. [OW83, Rud90, Lin01]). We expect that there is a proof more directly using the ShannonMcMillan-Breiman theorem (or related measure theoretic ideas) although we have not found it so far.

3.3. Step 3: Proof of $\overline{\operatorname{rdim}}\left(\mathcal{X}, \sigma_{1}, d, \mu\right) \leq 2 h_{\mu}\left(\mathcal{X}, \sigma_{1}, \sigma_{2}\right) / \log \alpha$. Let $X$ be a random variable taking values in $\mathcal{X}$ and obeying $\mu$. Let $0<\varepsilon<1$ and take $M>0$ with $\alpha^{-M}<\varepsilon \leq \alpha^{-M+1}$ as in Step 1. Let $N>0$. For each point $x \in \pi_{(-M, N+M) \times(-M, M)}(\mathcal{X})$ we choose $q(x) \in \mathcal{X}$ with $\pi_{(-M, N+M) \times(-M, M)}(q(x))=x$. Set $X^{\prime}=q\left(\pi_{(-M, N+M) \times(-M, M)}(X)\right)$ and $Y=\left(X^{\prime}, \sigma_{1} X^{\prime}, \sigma_{1}^{2} X^{\prime}, \ldots, \sigma_{1}^{N-1} X^{\prime}\right)$. Then

$$
\begin{gathered}
\frac{1}{N} \sum_{n=0}^{N-1} d\left(\sigma_{1}^{n} X, Y_{n}\right)=\frac{1}{N} \sum_{n=0}^{N-1} d\left(\sigma_{1}^{n} X, \sigma_{1}^{n} X^{\prime}\right) \leq \alpha^{-M}<\varepsilon . \\
I(X ; Y) \leq H(Y)=H\left(X^{\prime}\right)=H\left\{\left(X_{u}\right)_{u \in(-M, N+M) \times(-M, M)}\right\} .
\end{gathered}
$$

So

$$
\begin{gathered}
R(d, \mu, \varepsilon) \leq \frac{I(X ; Y)}{N} \leq \frac{1}{N} H\left\{\left(X_{u}\right)_{u \in(-M, N+M) \times(-M, M)}\right\}, \\
\frac{R(d, \mu, \varepsilon)}{\log (1 / \varepsilon)} \leq \frac{2 M}{\log (1 / \varepsilon)} \cdot \frac{1}{2 N M} H\left\{\left(X_{u}\right)_{u \in(-M, N+M) \times(-M, M)}\right\} .
\end{gathered}
$$

We first take the limit with respect to $N$ and next the limit with respect to $\varepsilon$. Noting $M / \log (1 / \varepsilon) \rightarrow 1 / \log \alpha$, we get

$$
\overline{\operatorname{rdim}}\left(\mathcal{X}, \sigma_{1}, d, \mu\right) \leq \frac{2 h_{\mu}\left(\mathcal{X}, \sigma_{1}, \sigma_{2}\right)}{\log \alpha} .
$$

3.4. Step 4: Proof of $\underline{\operatorname{rdim}}\left(\mathcal{X}, \sigma_{1}, d, \mu\right) \geq 2 h_{\mu}\left(\mathcal{X}, \sigma_{1}, \sigma_{2}\right) / \log \alpha$. We need the following lemma.

Lemma 3.7. Let $N \geq 1$ and $B$ a finite set. Let $X=\left(X_{0}, \ldots, X_{N-1}\right)$ and $Y=$ $\left(Y_{0}, \ldots, Y_{N-1}\right)$ be random variables taking values in $B^{N}$ (namely, each $X_{n}$ and $Y_{n}$ takes values in $B$ ) such that for some $0<\delta<1 / 2$

$$
\left.\mathbb{E} \text { (the number of } 0 \leq n<N \text { with } X_{n} \neq Y_{n}\right)<\delta N \text {. }
$$

Then

$$
I(X ; Y)>H(X)-N H(\delta)-\delta N \log |B|,
$$

where $H(\delta)=-\delta \log \delta-(1-\delta) \log (1-\delta)$ as in Step 2 . 
Proof. The proof is close to [LT18, Lemma 17]. Let $Z_{n}=1_{\left\{X_{n} \neq Y_{n}\right\}}$ and $Z=\{0 \leq n<$ $\left.N \mid X_{n} \neq Y_{n}\right\}$. We can identify $Z$ with $\left(Z_{0}, \ldots, Z_{N-1}\right)$ and hence

$$
\begin{aligned}
H(Z) & \leq H\left(Z_{0}\right)+\cdots+H\left(Z_{N-1}\right) \\
& =H\left(\mathbb{E} Z_{0}\right)+\cdots+H\left(\mathbb{E} Z_{N-1}\right) \\
& \leq N H\left(\frac{1}{N} \sum_{n=0}^{N-1} \mathbb{E} Z_{n}\right) \quad(\text { by concavity of } H(\cdot)) \\
& <N H(\delta) .
\end{aligned}
$$

So $H(Z)<N H(\delta)$. We decompose $H(X, Z \mid Y)$ in two ways:

$$
H(X, Z \mid Y)=H(X \mid Y)+H(Z \mid X, Y)=H(Z \mid Y)+H(X \mid Y, Z)
$$

$H(Z \mid X, Y)=0$ because $Z$ is determined by $X$ and $Y$. Hence

$$
H(X \mid Y)=H(Z \mid Y)+H(X \mid Y, Z)<N H(\delta)+H(X \mid Y, Z)
$$

We estimate

$$
H(X \mid Y, Z)=\sum_{E \subset\{0,1, \ldots, N-1\}} \mathbb{P}(Z=E) H(X \mid Y, Z=E) .
$$

Given $Y$ and the condition $Z=E$, the possibilities of $X$ is at most $|B|^{|E|}$. Therefore $H(X \mid Y, Z=E) \leq|E| \log |B|$ and

$$
\begin{aligned}
H(X \mid Y, Z) & \leq \sum_{E \subset\{0,1, \ldots, N-1\}}|E| \cdot \mathbb{P}(Z=E) \log |B| \\
& =\mathbb{E}|Z| \cdot \log |B| \\
& \leq \delta N \log |B| .
\end{aligned}
$$

As a conclusion, $H(X \mid Y)<N H(\delta)+\delta N \log |B|$ and $I(X ; Y)=H(X)-H(X \mid Y)>$ $H(X)-N H(\delta)-\delta N \log |B|$.

Let $X$ be a random variable taking values in $\mathcal{X}$ with $\operatorname{Law}(X)=\mu$ as in Step 3. Let $0<\varepsilon<\delta<1 / 2$ and $N>0$. Let $Y=\left(Y_{0}, \ldots, Y_{N-1}\right)$ be a random variable taking values in $\mathcal{X}^{N}$ and satisfying

$$
\mathbb{E}\left(\frac{1}{N} \sum_{n=0}^{N-1} d\left(\sigma_{1}^{n} X, Y_{n}\right)\right)<\varepsilon
$$

We estimate $I(X ; Y)$ from below. Take $M \geq 0$ satisfying $\delta \alpha^{-M-1}<\varepsilon \leq \delta \alpha^{-M}$. For $0 \leq n<N$, we set

$$
X_{n}^{\prime}=\pi_{\{n\} \times[-M, M]}(X)=\left(X_{n, m}\right)_{-M \leq m \leq M}, \quad Y_{n}^{\prime}=\pi_{\{0\} \times[-M, M]}\left(Y_{n}\right)=\left(\left(Y_{n}\right)_{0, m}\right)_{-M \leq m \leq M} .
$$


If $X_{n}^{\prime} \neq Y_{n}^{\prime}$ for some $n$ then $d\left(\sigma_{1}^{n} X, Y_{n}\right) \geq \alpha^{-M}$. So $\mathbb{E} d\left(\sigma_{1}^{n} X, Y_{n}\right) \geq \alpha^{-M} \mathbb{P}\left(X_{n}^{\prime} \neq Y_{n}^{\prime}\right)$ and hence

$$
\begin{aligned}
\mathbb{E}\left(\text { the number of } 0 \leq n<N \text { with } X_{n}^{\prime} \neq Y_{n}^{\prime}\right) & =\sum_{n=0}^{N-1} \mathbb{P}\left(X_{n}^{\prime} \neq Y_{n}^{\prime}\right) \\
& \leq \alpha^{M} \mathbb{E}\left(\sum_{n=0}^{N-1} d\left(\sigma_{1}^{n} X, Y_{n}\right)\right) \\
& <\alpha^{M} \varepsilon N \leq \delta N .
\end{aligned}
$$

Apply Lemma 3.7 to $X_{n}^{\prime}$ and $Y_{n}^{\prime}$ with $B=A^{2 M+1}$ :

$$
\begin{aligned}
I\left(X_{0}^{\prime}, \ldots, X_{N-1}^{\prime} ; Y_{0}^{\prime}, \ldots, Y_{N-1}^{\prime}\right)> & H\left(X_{0}^{\prime}, \ldots, X_{N-1}^{\prime}\right) \\
& -N H(\delta)-\delta N(2 M+1) \log |A| .
\end{aligned}
$$

By the data-processing inequality (Lemma 2.3),

$$
I(X ; Y) \geq I\left(X_{0}^{\prime}, \ldots, X_{N-1}^{\prime} ; Y_{0}^{\prime}, \ldots, Y_{N-1}^{\prime}\right)
$$

Therefore

$$
\frac{I(X ; Y)}{N} \geq \frac{H\left\{\left(X_{u}\right)_{u \in[0, N) \times[-M, M]}\right\}}{N}-H(\delta)-\delta(2 M+1) \log |A| .
$$

This holds for any $N>0$. So

$$
\begin{aligned}
R(d, \mu, \varepsilon) & \geq \inf _{N>0} \frac{H\left\{\left(X_{u}\right)_{u \in[0, N) \times[-M, M]}\right\}}{N}-H(\delta)-\delta(2 M+1) \log |A| \\
& =\lim _{N \rightarrow \infty} \frac{H\left\{\left(X_{u}\right)_{u \in[0, N) \times[-M, M]}\right\}}{N}-H(\delta)-\delta(2 M+1) \log |A| .
\end{aligned}
$$

We divide this by $\log (1 / \varepsilon)$ and take the limit $\varepsilon \rightarrow 0$. Noting $\log (1 / \varepsilon)<\log (1 / \delta)+(M+$ 1) $\log \alpha$ (here $\delta$ has been fixed), we get

$$
\underline{\operatorname{rdim}}\left(\mathcal{X}, \sigma_{1}, d, \mu\right) \geq \frac{2 h_{\mu}\left(\mathcal{X}, \sigma_{1}, \sigma_{2}\right)}{\log \alpha}-\frac{2 \delta \log |A|}{\log \alpha}
$$

Here we have used

$$
h_{\mu}\left(\mathcal{X}, \sigma_{1}, \sigma_{2}\right)=\lim _{N, M \rightarrow \infty} \frac{H\left\{\left(X_{u}\right)_{u \in[0, N) \times[-M, M]}\right\}}{N(2 M+1)} .
$$

Take the limit $\delta \rightarrow 0$. We get $\underline{\operatorname{rdim}}\left(\mathcal{X}, \sigma_{1}, d, \mu\right) \geq 2 h_{\mu}\left(\mathcal{X}, \sigma_{1}, \sigma_{2}\right) / \log \alpha$.

\section{REFERENCES}

[CT06] T. M. Cover, J. A. Thomas, Elements of information theory, second edition, Wiley, New York, 2006.

[ECG94] M. Effros, P. A. Chou, G. M. Gray, Variable-rate source coding theorems for stationary nonergodic sources, IEEE Trans. Inf. Theory vol. 40, pp. 1920-1925, 1994.

[EW11] M. Einsiedler, T. Ward, Ergodic theory with a view towards number theory, Graduate Texts in Mathematics 259, Springer, London. 
[Fur67] H. Furstenberg, Disjointness in ergodic theory, minimal sets, and a problem in Diophantine approximation, Math. Systems Theory 1 (1967) 1-49.

[Gra90] R.M. Gray, Entropy and information theory, New York, Springer-Verlag, 1990.

[Gro99] M. Gromov, Topological invariants of dynamical systems and spaces of holomorphic maps: I, Math. Phys. Anal. Geom. vol. 2 pp. 323-415, 1999.

[Gut15] Y. Gutman, Mean dimension and Jaworski-type theorems, Proceedings of the London Mathematical Society 111(4) (2015) 831-850.

[GLT16] Y. Gutman, E. Lindenstrauss, M. Tsukamoto, Mean dimension of $\mathbb{Z}^{k}$-actions, Geom. Funct. Anal. 26 Issue 3 (2016) 778-817.

[GQT] Y. Gutman, Y. Qiao, M. Tsukamoto, Application of signal analysis to the embedding problem of $\mathbb{Z}^{k}$-actions, arXiv:1709.00125, to appear in Geom. Funct. Anal.

[GT] Y. Gutman, M. Tsukamoto, Embedding minimal dynamical systems into Hilbert cubes, preprint, arXiv:1511.01802.

[KD94] T. Kawabata and A. Dembo, The rate distortion dimension of sets and measures, IEEE Trans. Inf. Theory, vol. 40, no. 5, pp. 1564-1572, Sep. 1994.

[LDN79] A. Leon-Garcia, L. D. Davisson, D. L. Neuhoff, New results on coding of stationary nonergodic sources, IEEE Trans. Inform. Theory, vol. 25, pp. 137-144, 1979.

[LL18] H. Li, B. Liang, Mean dimension, mean rank and von Neumann-Lück rank, J. Reine Angew. Math. 739 (2018) 207-240.

[Lin99] E. Lindenstrauss, Mean dimension, small entropy factors and an embedding theorem, Inst. Hautes Études Sci. Publ. Math. vol. 89 pp. 227-262, 1999.

[Lin01] E. Lindenstrauss, Pointwise theorems for amenable groups, Invent. math. 146 (2001) 259-296.

[LT18] E. Lindenstrauss, M. Tsukamoto, From rate distortion theory to metric mean dimension: variational principle, IEEE Trans. Inf. Theory, vol. 64, No. 5, pp. 3590-3609, May, 2018.

[LT19] E. Lindenstrauss, M. Tsukamoto, Double variational principle for mean dimension, Geom. Funct. Anal. 29 (2019) 1048-1109.

[LW00] E. Lindenstrauss, B. Weiss, Mean topological dimension, Israel J. Math. vol. 115 pp. 1-24, 2000.

[Ma79] R. Mañé, Expansive homeomorphisms and topological dimension, Trans. Amer. Math. Soc. 252 (1979) 313-319.

[Mat95] P. Mattila, Geometry of sets and measures in Euclidean spaces, Fractals and rectifiability, Cambridge Studies in Advanced Mathematics, 44, Cambridge University Press, Cambridge, 1995.

[MT19] T. Meyerovitch, M. Tsukamoto, Expansive multiparameter actions and mean dimension, Trans. Amer. Math. Soc. 371 (2019), 7275-7299

[OW83] D. S. Ornstein, B. Weiss, The Shannon-McMillan-Breiman theorem for a class of amenable groups, Israel J. Math. 44 (1983) 53-60.

[Rén59] A. Rényi, On the dimension and entropy of probability distributions, Acta Math. Sci. Hung. vol. 10, pp. 193-215, 1959.

[Rud90] D. J. Rudolph, Fundamentals of measurable dynamics, Clarendon Press, Oxford, 1990.

[Sh48] C. E. Shannon, A mathematical theory of communication, Bell Syst. Tech. J. 27 (1948) 379-423, 623-656.

[Sh59] C. E. Shannon, Coding theorems for a discrete source with a fidelity criterion, IRE Nat. Conv. Rec., Pt. 4, pp. 142-163, 1959.

[Tsu18] M. Tsukamoto, Mean dimension of the dynamical system of Brody curves, Invent. math. 211 (2018) 935-968. 
[Youn82] L.-S. Young, Dimension, entropy and Lyapunov exponents, Ergodic Theory Dynam. Systems 2 (1982) 109-124.

Mao Shinoda

Department of Human Coexistence, Graduate School of Human and Environmental Studies, Kyoto University, Yoshida-Nihonmaths-cho, Sakyo-ku, Kyoto, 606-8501, Japan Email: shinoda-mao@keio.jp

Masaki Tsukamoto

Department of Mathematics, Kyushu University, Moto-oka 744, Nishi-ku, Fukuoka 8190395, Japan

E-mail: masaki.tsukamoto@gmail.com 